\title{
Environmental stability study of holographic solar spectrum splitting materials
}

\author{
Benjamin D. Chrysler ${ }^{* a}$, Silvana Ayala Pelaez ${ }^{\mathrm{a}}$, Yuechen Wu ${ }^{\mathrm{a}}$, Shelby D. Vorndran ${ }^{\mathrm{b}}$, Raymond K. \\ Kostuk $^{\mathrm{a}, \mathrm{b}}$ \\ ${ }^{a}$ Electrical and Computer Engineering Dept., University of Arizona, 1230 E Speedway Blvd., \\ Tucson, AZ USA 85721; ${ }^{\mathrm{b}}$ College of Optical Sciences, University of Arizona, 1630 E University \\ Blvd., Tucson, AZ USA 85721;
}

\begin{abstract}
In this study the impact of outdoor temperature variations and solar illumination exposure on spectral filter material and holographic optical elements is examined. Although holographic components have been shown to be useful for solar spectrum splitting designs, relatively little quantitative data exist to demonstrate the extent to which these materials can withstand outdoor conditions. As researchers seek to investigate practical spectrum splitting designs, the environmental stability of holographic materials should be considered as an important factor. In the experiment presented, two holographic materials, Covestro Bayfol HX photopolymer and dichromated gelatin, and 3M reflective polymer filter materials are exposed to outdoor conditions for a period of several months. The environmental effect on absorption, spectral and angular bandwidth, peak efficiency, and Bragg matching conditions for the holograms are examined. Spectral bandwidth and transmittance of the $3 \mathrm{M}$ reflective filter material are also monitored. Holographic gratings are recorded, measured, and mounted on glass substrates and then sealed with a glass cover plate. The test samples are then mounted on a photovoltaic panel to simulate realistic temperature conditions and placed at an outdoor test facility in Tucson, Arizona. A duplicate set of holograms and $3 \mathrm{M}$ filter material is stored as a control group and periodically compared over the test period.
\end{abstract}

Keywords: spectrum splitting, holographic optical elements, photovoltaics, dichromated gelatin, Covestro Bayfol HX, $3 \mathrm{M}$ dichroic, environmental stability, degradation

\section{INTRODUCTION}

One approach to achieving high efficiency photovoltaic (PV) modules is to split the incident solar energy spectrum between PV cells with different bandgap energies. Spectrum Splitting Systems (SSS) achieve this by directing spectral bands of solar power to spatially separated single-junction cells. This technique allows individual cells to be chosen based on the improvement that results from using combinations of cells that most effectively utilize the available incident solar insolation as determined by the detailed balance limit ${ }^{1}$. In addition, the geometry and concentration ratio of spectrum splitting systems can be optimized to increase the collection of diffuse light, which accounts for more than $20 \%$ of total solar insolation even in sunny regions such as Tucson, $\mathrm{AZ}^{2}$.

Numerous SSS designs using holographic ${ }^{3-6}$ or dichroic ${ }^{7-10}$ optical filters to spatially isolate solar spectral bands have been proposed. Holographic optical elements (HOE) are a versatile, inexpensive option for SSS, which can: achieve high diffraction efficiency, be used in transmission or reflection geometries, incorporate concentration, and be customized for individual designs by modifying diffracted spectral bands and dispersion characteristics ${ }^{11-14}$. Dichroic reflection filters allow for simple optical designs and can achieve high bandwidth, low polarization sensitivity, and nearly ideal spectral filtering properties ${ }^{15}$.

*bchrysler@email.arizona.edu

Next Generation Technologies for Solar Energy Conversion VII, edited by Oleg V. Sulima,

Gavin Conibeer, Proc of SPIE Vol 9937, 99370N - (C) 2016 SPIE

CCC code: $0277-786 \mathrm{X} / 16 / \$ 18 \cdot$ doi: $10.1117 / 12.2237071$

Proc. of SPIE Vol. $993799370 \mathrm{~N}-1$ 
Dichromated gelatin (DCG) is the most common holographic material proposed for SSS designs. It consists of a dehydrated gelatinous matrix interspersed with an ammonium dichromate sensitizer. When DCG is exposed to an interference pattern of light, the high intensity regions are selectively hardened, and a series of chemical processes results in refractive index modulation. DCG has a tunable bandwidth of over $300 \mathrm{~nm}$, high diffraction efficiency, and low scattering properties which make it an attractive holographic material ${ }^{16}$.

Covestro Bayfol HX is a photopolymer that has been developed in the last several years and is being increasingly used for various HOE applications ${ }^{17}$. Bayfol HX has recently been suggested for use in spectrum splitting systems, due to its high index modulation, suitable bandwidth of $230 \mathrm{~nm}$, and 1-step dry processing photobleaching process ${ }^{18}$.

Dichroic reflecting filters are produced in a relatively low-cost, multi-layer polymeric film by companies such as $3 \mathrm{M}^{19}$. The films have primarily been used for architectural and aesthetic purposes, but also have attractive optical characteristics for $\mathrm{SSS}^{20}$.

Materials used in PV modules must be highly durable, with reliable lifespans over 30 years under harsh conditions in order to maintain acceptable module efficiency. PV panel surface temperatures can exceed $80^{\circ} \mathrm{C}$ in summer months and daily temperature variations of $30^{\circ} \mathrm{C}$ being common in many areas of the continental U.S. These variations result in high mechanical stress levels on the materials. Cumulative UV radiation also degrades many polymers and encapsulating materials $^{21,22}$.

There is little or no published data regarding environmental stability of candidate holographic and dichroic polymer materials for SSS applications. This information should be a key design consideration for researchers working on SSS. Materials that cannot withstand realistic PV conditions over many years will not make a practical choice for SSS, regardless of the positive characteristics of the material in laboratory conditions and also points the way for new research on packaging materials.

This study examines optical performance of DCG, Covestro Bayfol HX, and 3M dichroic films over 16 weeks of sun exposure in Tucson, AZ.

\section{EXPERIMENTAL SETUP}

Three materials were selected for the study based on their suitability for spectrum splitting. DCG was prepared using a mold coating procedure resulting in 20um thick film. Covestro Bayfol HX 102 (Covestro is formerly Bayer MaterialScience $)^{23}$ is a commercially available photopolymer film with 16 um thick film. 3M DF-PA Chill and DF-PA Blaze dichroic filters ${ }^{24}$ were selected, with Chill filters reflecting $500 \mathrm{~nm}-615 \mathrm{~nm}$ and Blaze filters reflecting $590 \mathrm{~nm}-$ $795 \mathrm{~nm}$.

DCG reflection holograms in this study are unslanted gratings formed with construction angles of $10^{\circ} / 170^{\circ}$ relative to the film normal and exposed with $650 \mathrm{~mJ} / \mathrm{cm}^{2}$ of $532 \mathrm{~nm}$ light. After processing they had sharp spectral filter transitions and a wide bandwidth of $135 \mathrm{~nm}$. Bayfol HX reflection holograms in this study are unslanted gratings formed with construction angles of $20^{\circ} / 160^{\circ}$ and exposed with $200 \mathrm{~mJ} / \mathrm{cm}^{2}$ of $532 \mathrm{~nm}$ light. After processing they had a narrow bandwidth of $18 \mathrm{~nm}$.

The DCG transmission holograms in this study are slanted gratings formed with construction angles of $-20^{\circ} / 5^{\circ}$ and exposed with $250 \mathrm{~mJ} / \mathrm{cm}^{2}$ at $532 \mathrm{~nm}$. Bayfol HX transmission holograms are slanted gratings formed with construction angles of $-5^{\circ} / 15^{\circ}$ and exposed with $24 \mathrm{~mJ} / \mathrm{cm}^{2}$ at $532 \mathrm{~nm}$. After processing, the Bragg angles were $-5^{\circ} / 15^{\circ}$ for Bayfol $\mathrm{HX}$ and $-20^{\circ} / 0^{\circ}$ for DCG.

Each sample was encapsulated and sealed with Sylgard Silicon Elastomer 184 and a cover glass for protection against water vapor, precipitation, and abrasive particles, while maintaining optical transparency. The glue is intended for use as an encapsulant for PV modules, and is hydrophobic and water resistant. No visible degradation of the seal was observed over the course of the study. Note that although this might not be an ideal encapsulating method since the sealant was not 
applied under vacuum, all samples were prepared in the same way and provide a common point for comparison. An example of a sealed hologram is shown in Fig. 1a.

Two identical groups of each material sample were prepared: one for exposure and one as a control group. Each group (control and exposure) consists of a total of 12 test samples, consisting of two transmission and two reflection holograms in DCG and Bayfol HX, one 3M Blaze filter, one 3M Chill filter, and two samples with no spectral filter material, which are used to observe any changes that may occur to the encapsulant material.

The experimental group was attached to a silicon photovoltaic panel as shown in Fig. $1 \mathrm{~b}$ and placed in an unobstructed area in Tucson, AZ to receive solar insolation. The panel was southward facing and titled at approximately $30^{\circ}$ corresponding to the latitude angle of Tucson, AZ. The control group was placed in a dark cabinet in a climate controlled building, free from thermal or radiation stresses.

The experimental group was exposed to solar illumination for periods of four weeks at a time and then the optical properties of the holograms and filter material were experimentally measured. The cover glass and substrate for each sample was cleaned before measuring to eliminate differences due to dirt on the exposed samples.

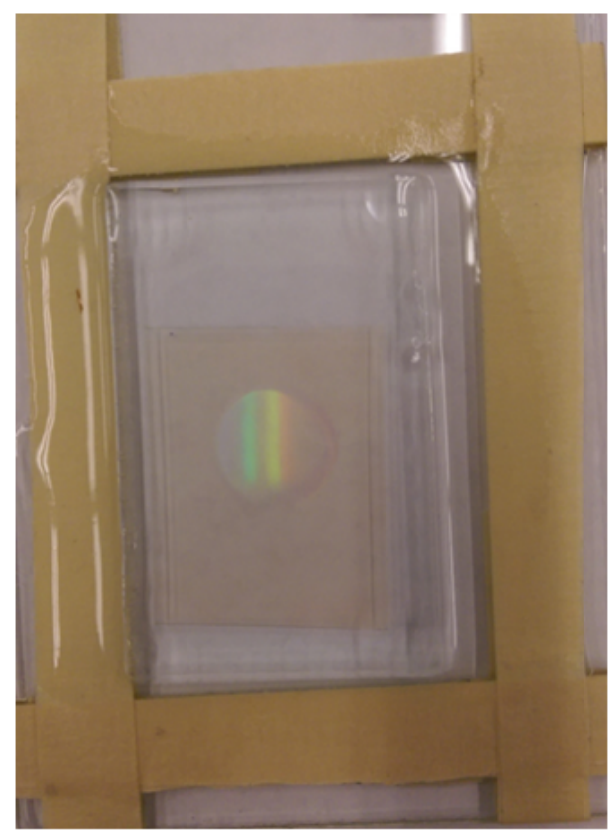

(a)

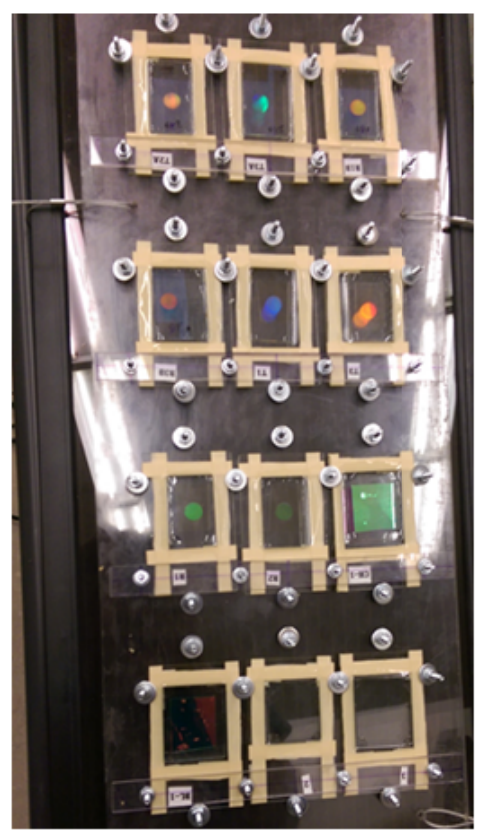

(b)

Figure 1: (a) Sealed Bayfol HX transmission hologram sample. (b) Experimental group samples attached to PV panel.

\section{PERFORMANCE METRICS}

An ideal spectral filter directs all spectral components of incident solar energy to the PV cell that most efficiently converts it to electrical power. Russo et al described filter performance in terms of the in-band and out-of-band spectral regions ${ }^{14}$. The holographic filter is an approximation to the ideal spectral filter and is designed to diffract light within the in-band spectral region while transmitting light in the out-of-band spectral region. The following grating characteristics are measured over the course of this study because of their direct impact on the filter performance:

- Diffraction efficiency

- Spectral bandwidth 
- Bragg angle

- Absorption and scattering

A spectrum splitting holographic filter should have high diffraction efficiency over the desired spectral bandwidth and direct light to the optimal PV cell at the designed Bragg angle. Deviations from the designed grating characteristics result in fewer photons being directed to the appropriate PV cell and a reduction in system efficiency.

The optical materials used for SSS should have low absorption and scattering. Absorbed energy is thermalized and results in an unrecoverable loss in available solar energy and increased system temperature. Scattering also introduces system loss, as backscattered light is unrecoverable and forward scattered light may not be absorbed by the PV cell.

The exposed holograms are evaluated by measuring the diffraction efficiency as a function of angle of incidence at the recording wavelength of 532nm for the transmission holograms and the reflection holograms recorded in the Bayfol HX material. Reflection holograms recorded in DCG were measured at $632.8 \mathrm{~nm}$ to reduce the angle of incidence during measurements. The diffraction efficiency $\eta$ as a function of angle ( $\theta$ relative to the hologram normal) is obtained from the following ratio:

$$
\eta(\theta)=\frac{P_{\text {diff }}(\theta)}{P_{\text {inc }}-P_{\text {refl }}(\theta)},
$$

Where $P_{\text {diff }}$ is the measured optical power of the diffracted beam with incident power $P_{\text {inc }}$, and $P_{\text {refl }}$ is the power reflected from the surface of the hologram.

Spectral transmittance measurements were used to indicate the diffraction efficiency of the holograms as a function of wavelength. The spectral transmittance $\mathrm{T}$, was obtained by illuminating the hologram at the Bragg angle of incidence and measuring the transmitted spectrum according to:

$$
T(\lambda)=\frac{P_{\text {trans }}(\lambda) / P_{\text {inc }}(\lambda)}{1-P_{\text {refl }}(\lambda) / P_{\text {inc }}(\lambda)},
$$

where $P_{\text {trans }}$ is the transmitted power as a function of wavelength $(\lambda), P_{\text {inc }}$ is the power incident at wavelength $\lambda$, and $P_{\text {refl }}$ is the power reflected from the surface of the cover glass at each wavelength.

The spectral transmittance of the glass and the Fresnel reflected light were accounted for in the hologram transmittance values. A tungsten halogen lamp was used for the optical source and a fiber-coupled Ocean Optics spectrometer (model USB2000+) with a resolution of $4 \mathrm{~nm}$ was used for the spectral power measurements. The transmittance of the holographic film was also measured where no grating was present to determine the absorption and scattering levels present in the film alone.

Spectral transmittance measurements of the $3 \mathrm{M}$ dichroic film were evaluated by comparing the transmittance of the inband and out-of-band regions of the dichroic filter after each exposure period. Fresnel reflection and glass transmittance were also taken into account for the $3 \mathrm{M}$ filter measurements.

No discernable changes in measured characteristics or visual appearance were detected for any of the samples that were kept indoors.

\section{MEASUREMENT RESULTS}

\subsection{Covestro Bayfol HX}

The transmittance spectrum of Bayfol HX film in regions with no grating measured after each 4-week exposure is shown in Fig. 2a, where each curve is the average of the four exposed samples for the respective measurement period. A consistent reduction in film transmittance was observed over the entire spectral range for each sample. The reduction is 
attributed to an increase in absorption and a distinct yellowing of film was observed. The increase in absorption is much larger at shorter wavelengths. After 16 weeks of sun exposure, a $43 \%$ increase in absorption was measured at $400 \mathrm{~nm}$, and a $6 \%$ increase in absorption was measured at $800 \mathrm{~nm}$ as determined from the transmittance measurements.

Diffraction efficiency curves for selected Bayfol HX transmission and reflection holograms over the course of the study are shown in Figs. $2 \mathrm{~b}$ and $2 \mathrm{c}$. The results for one of the measured transmission and reflection holograms are included in Figs. $2 \mathrm{~b}$ and $2 \mathrm{c}$ to illustrate the main characteristics, however an average of both exposed samples of each type are used for the performance values given in table 1 . The average reduction in peak diffraction efficiency for the transmission holograms was $22 \%$ and $26 \%$ for reflection holograms. No changes in Bragg angle for transmission holograms were detected, but a $6^{\circ}$ degree change in Bragg angle for the reflection holograms corresponding to material swelling was measured.

Spectral transmittance for the reflection hologram is shown in Fig. 2d. In addition to reduced diffraction efficiency after exposure, the figure shows a spectral bandwidth increase of $56 \%$ and a distorted filter profile. The peak diffracted wavelength increased by $7.9 \mathrm{~nm}$, corresponding to a material swelling of $1.5 \%$. This value is also consistent with swelling calculated from the Bragg angle shift.

Visual inspection of the reflection holograms after week 16 showed the color of the grating to vary from green and blue when viewed at normal incidence. This indicates that the reported changes in diffraction efficiency, spectral bandwidth, and film swelling are not uniform over the grating aperture.

Air pockets were also observed to form around the edges of the film, expanding inward over time. The pockets may have formed due to microscopic air pockets present around the edges of the film during the sealing process. The air pockets were not present in regions of the hologram that were measured and did not directly affect the measurements. Measurements in the regions of Bayfol HX film in contact with the air pockets did not appear to be affected differently from regions without the air pockets.

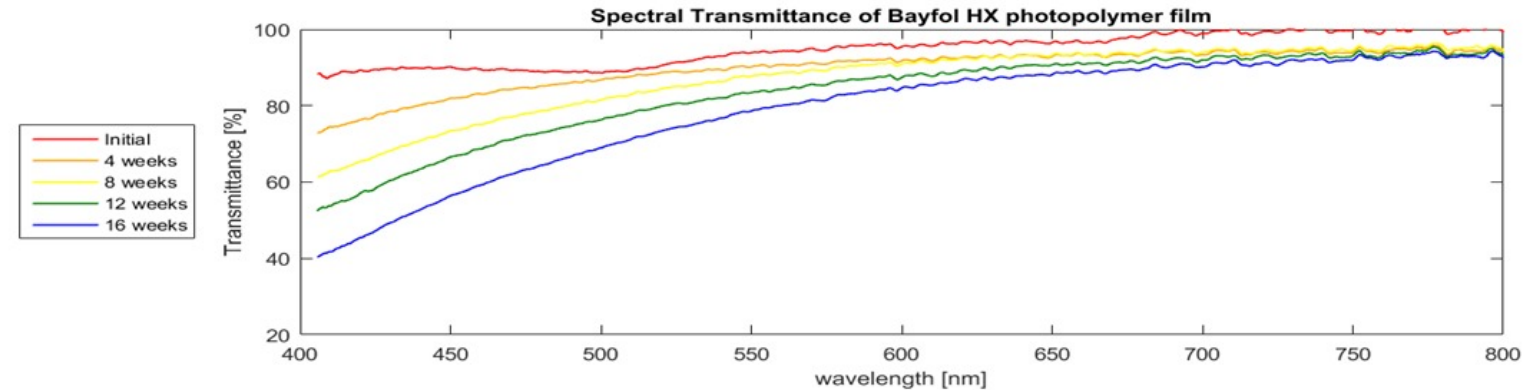

(a)

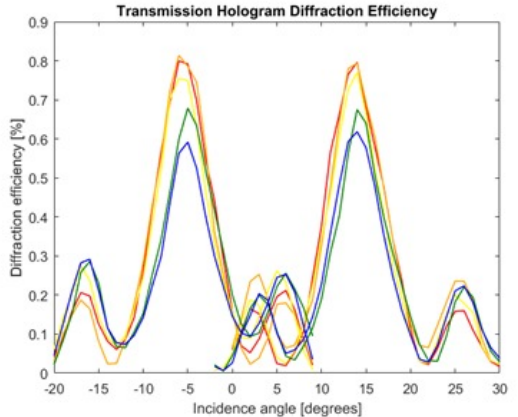

(b)

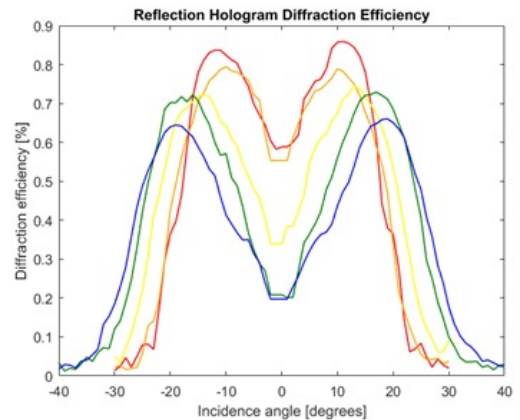

(c)

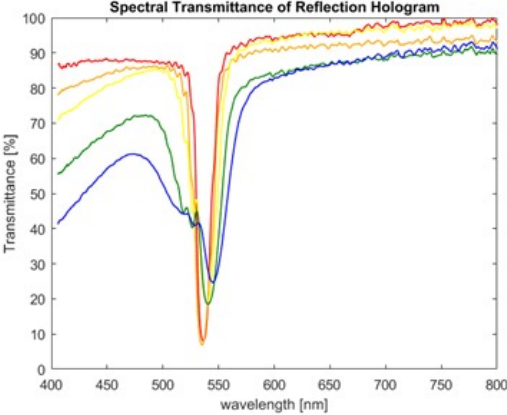

(d)

Figure 2: Each curve shows measurements of Bayfol HX photopolymer samples after 4-week intervals of sun exposure for a total of 16 weeks. (a) Spectral transmittance of the film. A strong increase in absorption is observed for the shorter wavelengths. (b)

Diffraction efficiency of the transmission hologram. Steady reduction in diffraction efficiency is observed. (c) Diffraction efficiency of the reflection hologram. Steady reduction in diffraction efficiency is observed, as well as a change in the Bragg angle. (d) Spectral transmission of the reflection hologram. Distortion of spectral band and shift toward longer wavelengths is observed. 


\subsection{Dichromated Gelatin Holograms}

No significant changes in material absorption or scattering were discernable for dichromated gelatin samples over 16 weeks of exposure.

Figs. $3 a$ and $3 b$ show the diffraction efficiency curves for one of the DCG transmission and reflection hologram samples. Only one of the measured transmission and reflection holograms are included in Figs. 3a and $3 \mathrm{~b}$ as the duplicate hologram has very similar characteristics, however, an average of both exposed samples of each type are used for the performance metrics. The transmission holograms showed a slight $(3 \%)$ reduction in peak diffraction efficiency over the 16 weeks of exposure and the reflection holograms also showed a slight reduction in peak diffraction efficiency of $2 \%$.

The reduction of angular bandwidth shown in Fig. $3 \mathrm{~b}$ is the result of film shrinkage. This can also be determined from the spectral transmittance curve in Fig. 3c, where the diffracted spectral band shifts to shorter wavelengths. The average shift for the reflection holograms is $27 \mathrm{~nm}$, corresponding to $3 \%$ film shrinkage, which is consistent with the level of shrinking that the reduction in angular bandwidth indicates.

Similar to the Bayfol HX samples, air pockets were observed to form near the film boundary in DCG. In the DCG samples, film cloudiness due to scatter in unexposed areas of the sample decreased in regions in contact with the air pockets. The air pockets were not present in regions of the hologram that were measured and did not directly affect the measurements.

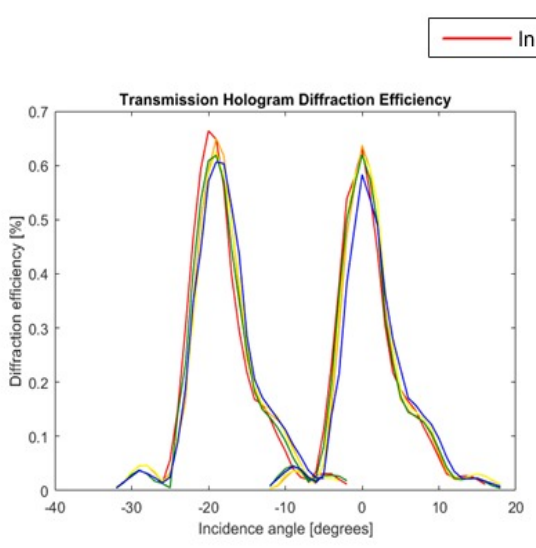

(a)

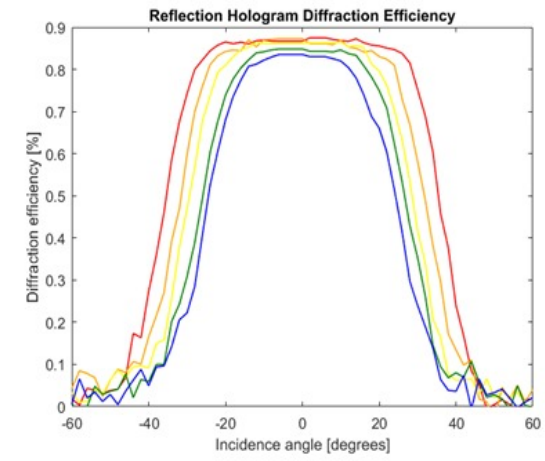

(b)

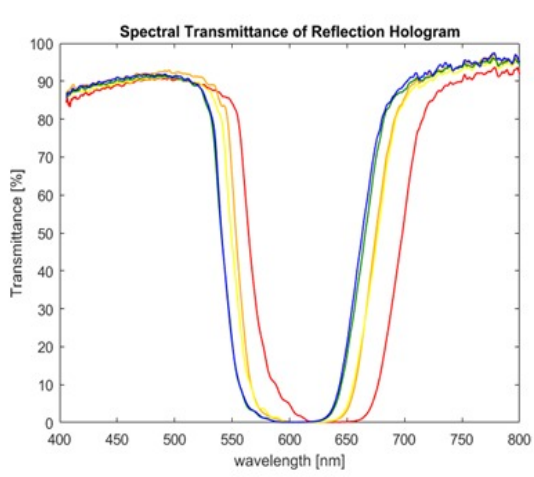

(c)

Figure 3: Each curve shows measurements of DCG samples after 4-week intervals of sun exposure for a total of 16 weeks. (a) Diffraction efficiency of the transmission hologram. Slight reduction in diffraction efficiency is observed. (b) Diffraction efficiency of the reflection hologram. Slight reduction in diffraction efficiency is observed, as well as change in Bragg angles. (c) Spectral transmission of the reflection hologram. A shift toward shorter wavelengths and slight reduction in bandwidth are observed.

\subsection{M Dichroic Film}

The spectral transmittance for the exposed 3M blaze film and 3M chill film are shown in Figs. 4a and 4b. No discernable degradation in either $3 \mathrm{M}$ film occurred over the 16 weeks of exposure. Overall, the $3 \mathrm{M}$ film seemed to perform the best of the three materials, with no significant measured change in transmittance and no formation of air pockets in the 
laminated samples. The small variations observed in the $3 \mathrm{M}$ chill film do not follow a consistent pattern and may be due to noise in the measurement process.

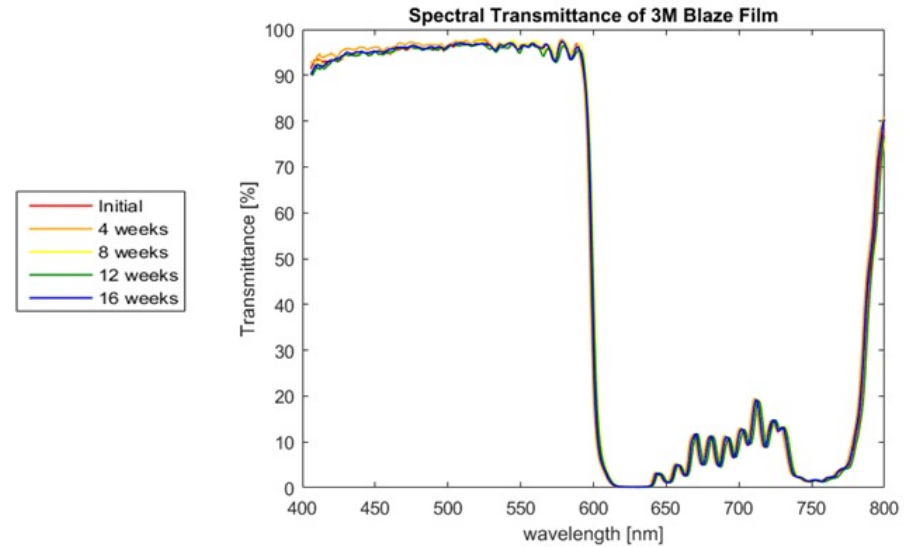

(a)

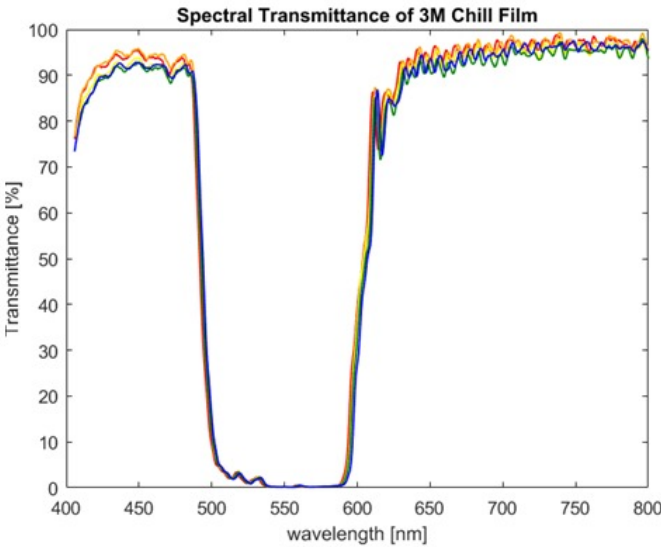

(b)

Figure 4: Each curve shows spectral transmittance measurements of 3M film samples after 4-week intervals of sun exposure for a total of 16 weeks. (a) $3 \mathrm{M}$ Blaze dichroic film. (b) $3 \mathrm{M}$ chill dichroic film.

\subsection{Summary}

A summary of the measured degradation for each material is tabulated in Table 1. A dash '-" indicates that no discernable changes are reported, and a gray filled in box indicates that measurement was not taken or is not applicable for the given material type.The values are an average of the results from both exposed samples.

Table 1: Measured changes in optical performance of Bayfol HX, DCG, and 3M Blaze and Chill dichroic films. A dash '-' indicates that no discernable changes are reported, and a gray filled in box indicates that measurement was not taken or is not applicable for the given material type.

\begin{tabular}{|c|c|c|c|c|c|c|c|}
\hline & & $\begin{array}{c}\text { Diffraction } \\
\text { Efficiency } \\
{[\%,} \\
\text { absolute] }\end{array}$ & $\begin{array}{c}\text { Spectral } \\
\text { Bandwidth } \\
{[\%,} \\
\text { relative] }\end{array}$ & $\begin{array}{l}\text { Bragg } \\
\text { Angle } \\
\text { [degrees } \\
\text { ] }\end{array}$ & $\begin{array}{c}\text { Absorption } \\
\text { (400nm) } \\
{[\%,} \\
\text { absolute] }\end{array}$ & $\begin{array}{c}\text { Absorption } \\
\text { (800nm) } \\
{[\%,} \\
\text { absolute] }\end{array}$ & $\begin{array}{l}\text { Film } \\
\text { Thickness } \\
{[\%,} \\
\text { relative] }\end{array}$ \\
\hline \multirow{2}{*}{$\begin{array}{c}\text { Bayfol } \\
\text { HX }\end{array}$} & Transmission & $-22 \%$ & & - & \multirow{2}{*}{$+43 \%$} & \multirow{2}{*}{$+6 \%$} & \multirow{2}{*}{$+1.5 \%$} \\
\hline & Reflection & $-26 \%$ & $+56 \%$ & $+6^{\circ}$ & & & \\
\hline \multirow{2}{*}{ DCG } & Transmission & $-3 \%$ & & - & \multirow{2}{*}{ - } & \multirow{2}{*}{ - } & \multirow{2}{*}{$-4.5 \%$} \\
\hline & Reflection & $-2 \%$ & $-7 \%$ & & & & \\
\hline \multicolumn{2}{|c|}{ 3M Dichroic } & & - & & - & - & - \\
\hline
\end{tabular}




\section{CONCLUSION}

Bayfol HX, dichromated gelatin, and 3M dichroic filters were tested for environmental stability by measuring optical performance after exposing samples to realistic thermal and sun exposure conditions over 4-week intervals for a total exposure time of 16 weeks. Significant levels of degradation in diffraction efficiency, Bragg angle shift, and absorption are observed in Bayfol HX photopolymer. Less degradation in the performance parameters of DCG holograms were found, and no detectable degradation in performance parameters were measured for $3 \mathrm{M}$ dichroic film during the same solar exposure period.

Bayfol HX 102 does not seem to offer the environmental stability required for spectrum splitting applications. Dichromated gelatin seems to have suitable properties, but further investigation may be necessary to fully understand how film composition and the sealing process affect environmental stability. Dichroic polymeric films, similar to those available from $3 \mathrm{M}$, are promising and their long term stability will continue to be monitored for longer exposure periods.

\section{ACKNOWLEDGEMENTS}

The authors wish to acknowledge support from the NSF/DOE ERC cooperative agreement No. EEC-1041895, NSF grant ECCS-1405619

\section{REFERENCES}

[1] De Vos, Alexis, "Detailed balance limit of the efficiency of tandem solar cells", Journal of Physics D: Applied Physics 13(5), 839 (1980).

[2] "Solar Radiation Data Manual for Flat-Plate and Concentrating Collectors," NREL, $<$ http://rredc.nrel.gov/solar/pubs/redbook/> (28 August 2016).

[3] Collados, M. Victoria, Daniel Chemisana, and Jesús Atencia, "Holographic solar energy systems: The role of optical elements," Renewable and Sustainable Energy Reviews 59, 130-140 (2016).

[4] Russo, Juan M., Deming Zhang, Michael Gordon, Shelby D. Vorndran, Youchen Wu, and Raymond K. Kostuk, "Grating-over-lens concentrating photovoltaic spectrum splitting systems with volume holographic optical elements," Proc. SPIE 8821, 882106 (2013).

[5] Zhang, Deming, Michael Gordon, Juan M. Russo, Shelby Vorndran, and Raymond K. Kostuk. "Spectrum-splitting photovoltaic system using transmission holographic lenses," J. Photon. Energy. 3(1), 034597 (2013).

[6] Wu, Yuechen, Shelby D. Vorndran, Juan M. Russo, Silvana Ayala, and Raymond K. Kostuk, "Design of folded holographic spectrum-splitting photovoltaic system for direct and diffuse illumination conditions," Proc. SPIE 9175, 91750G (2014).

[7] Fraas, L. M., J. E. Avery, H. X. Huang, E. Shifman, K. Edmondson, and R. R. King, "Toward 40\% and higher solar cells in a new Cassegrainian PV module," In Conference Record of the Thirty-first IEEE Photovoltaic Specialists Conference, 751-753 (2005).

[8] Zhengshan, J. Yu, Kathryn C. Fisher, Brian M. Wheelwright, Roger P. Angel, and Zachary C. Holman, "PVMirror: a new concept for tandem solar cells and hybrid solar converters," IEEE Journal of Photovoltaics 5(6), 1791-1799 (2015).

[9] Vincenzi, Donato, Alessandro Busato, Marco Stefancich, and Giuliano Martinelli, "Concentrating PV system based on spectral separation of solar radiation," Physica Status Solidi (a) 206(2), 375-378 (2009).

[10] Barnett, Allen, Douglas Kirkpatrick, Christiana Honsberg, Duncan Moore, Mark Wanlass, Keith Emery, Richard Schwartz et al, "Very high efficiency solar cell modules," Progress in Photovoltaics: Research and Applications 17(1), 75-83 (2009). 
[11] Kogelnik, Herwig, "Coupled wave theory for thick hologram gratings," Bell System Technical Journal 48(9), 29092947 (1969).

[12] Zhang, Deming, Michael Gordon, Juan M. Russo, Shelby Vorndran, Matthew Escarra, Harry Atwater, and Raymond K. Kostuk, "Reflection hologram solar spectrum-splitting filters," Proc. SPIE 8468, 846807 (2012).

[13] Russo, Juan Manuel, Shelby Vorndran, Yuechen Wu, and Raymond K. Kostuk, "Cross-correlation analysis of dispersive spectrum splitting techniques for photovoltaic systems," J. Photon. Energy. 5(1), 054599 (2015).

[14] Russo, Juan M., Deming Zhang, Michael Gordon, Shelby Vorndran, Yuechen Wu, and Raymond K. Kostuk, "Spectrum splitting metrics and effect of filter characteristics on photovoltaic system performance," Optics express 22(102), A528-A541 (2014).

[15] Zhang, Deming, Yuechen Wu, Juan M. Russo, Michael Gordon, Shelby Vorndran, and Raymond K. Kostuk, "Optical performance of dichroic spectrum-splitting filters," J. Photon. Energy 4(1), 043095 (2014).

[16] Stojanoff, Christo G, "Review of the technology for the manufacturing of large-format DCG holograms for technical applications," Proc. SPIE 3011, 267-278 (1997).

[17] Jurbergs, David, Friedrich-Karl Bruder, Francois Deuber, Thomas Fäcke, Rainer Hagen, Dennis Hönel, Thomas Rölle, Marc-Stephan Weiser, and Andy Volkov, "New recording materials for the holographic industry," Proc. SPIE 7233, 72330K (2009).

[18] Marín-Sáez, Julia, Jesús Atencia, Daniel Chemisana, and María-Victoria Collados, "Characterization of volume holographic optical elements recorded in Bayfol HX photopolymer for solar photovoltaic applications," Optics express 24(6), A720-A730 (2016).

[19] Weber, Michael F., Carl A. Stover, Larry R. Gilbert, Timothy J. Nevitt, and Andrew J. Ouderkirk, "Giant birefringent optics in multilayer polymer mirrors," Science 287(5462), 2451-2456 (2000).

[20] Zhang, Deming, Yuechen Wu, Juan M. Russo, Michael Gordon, Shelby Vorndran, and Raymond K. Kostuk, "Optical performance of dichroic spectrum-splitting filters," J. Photon. Energy. 4(1), 043095 (2014).

[21] Miller, David C., and Sarah R. Kurtz, "Durability of Fresnel lenses: a review specific to the concentrating photovoltaic application," Solar Energy Materials and Solar Cells 95(8), 2037-2068 (2011).

[22] Czanderna, A. W., and F. J. Pern, "Encapsulation of PV modules using ethylene vinyl acetate copolymer as a pottant: A critical review," Solar energy materials and solar cells 43(2), 101-181 (1996).

[23] "Polycarbonate Blend Films and Special Films", Covestro AG, $<$ http://www.films.covestro.com/en/Products/Bayfol> (9 August 2016).

[24] "Dichroic Glass Finishes", 3M Solutions, 2016, <http://solutions.3m.com/wps/portal/3M/en_US/architecturalmarkets/home/glass-finishes/dichroic/> (9 August 2016). 